\title{
Distribución y susceptibilidad de cepas de Staphylococcus aureus aisladas de cinco comunidades en Bogotá, Colombia, 2013.
}

Francisco Javier Africano Díaz ${ }^{1}$, Iván Alberto Méndez Rodríguez ${ }^{2}$, Diego Fabián Holguín Riaño ${ }^{3}$ Diana Patricia Pachón Barinas ${ }^{4}$, Nydia Alexandra Rojas Ávila ${ }^{5}$.

\section{Resumen}

1. Médico General, Universidad Militar Nueva Granada.

2. MSc Microbiología, Profesor de la Facultad de Medicina, Universidad Militar Nueva Granada, Grupo de Investigación Patogenicidad Microbiana.

3. Médico General, Universidad Militar Nueva Granada.

4. MSc Microbiología, Profesor de la Facultad de Medicina, Universidad Militar Nueva Granada, Grupo de Investigación Patogenicidad Microbia$n a$.

5. MD, Cd MSc Salud Pública, Profesor de la Facultad de Medicina, Universidad Militar Nueva Granada.

Autor de correspondencia: Francisco Javier Africano Díaz, javihalo2@gmail.com

Como citar:Africano FJ, Méndez IA, Holguín DF, Pachón DP, Rojas NA. Distribución y susceptibilidad de cepas de Staphylococcus aureus aisladas de cinco comunidades en Bogotá, Colombia, 2013. Revista Cuarzo 2018:24 (2) 7-12.

Recibido: 18 de mayo de 2018. Aceptado: 13 de septiembre de 2018. Publicado: 30 de diciembre de 2018

Licencia creative commons (c) $($ ) $(9)$
Staphylococcus aureus ha sido reconocido como uno de los microorganismos con resistencia a los antimicrobianos y mayor impacto incluso en infecciones adquiridas en la comunidad.

Objetivo: El presente estudio pretende establecer la prevalencia y el perfil de susceptibilidad en aislamientos de Staphylococcus aureus de diferentes comunidades en la ciudad de Bogotá D.C., Colombia.

Materiales y Métodos: 150 muestras de 5 diferentes comunidades fueron obtenidas mediante hisopado nasal y procesadas para identificación del S. aureus y su perfil de susceptibilidad.

Resultados: De estudiantes de Medicina, amas de casa, personas de atención al público, manipuladores de alimentos y conductores de servicio público, se aislaron $43(28,7 \%)$ cepas de $\mathrm{S}$. aureus, 25 en mujeres $(58 \%)$ y 18 en hombres $(42 \%): 88,4 \%(38)$ se tipificaron como meticilino sensibles (SAMS) y 11,6\% (5) como meticilino resistente (SAMR). Se encontró resistencia a la clindamicina (31,6\% para los SAMS, 60\% para los SAMR); al linezolid (50\% en los SAMS, 80\% para los SAMR) y para el trimetoprim sulfametoxazol (18,9\% para los SAMS, 60\% para los SAMR).

Conclusión: Bajo nivel de cepas de SAMS (25,3\%) y SAMR (3,3\%) en las comunidades evaluadas, en contraste a estudios en trabajadores de la salud (prevalencia para SAMR del 30\%).

Palabras clave: Staphylococcus aureus, Comunidad, Staphylococcus aureus meticilino resistente

\section{Distribution and susceptibility of Staphylococcus au- reus strains isolated from five communities in Bogotá, Colombia, 2013}

\begin{abstract}
Staphylococcus aureus has been recognized as one of the microorganisms with antimicrobial resistance and even greater impact on community-acquired infections.
\end{abstract}

Objective: This study aims to establish the prevalence and susceptibility profile of Staphylococcus aureus isolates from different communities in the city of Bogota, Colombia.

Materials and Methods: 150 samples from 5 different communities were obtained by nasal swabs and processed for identification of $S$. aureus and its susceptibility profile.

Results: From medical students, housewives, people in customer service, food handlers and drivers of public service, we isolated $43(28,7 \%)$ strains of S. aureus, 25 from women (58\%) and 18 from men (42\%): 88.4\% (38) were typed as methicillin susceptible (MSSA) and 11.6\% (5) as methicillin-resistant (MRSA). Resistance was found to clindamycin (31,6\% for MSSA, $60 \%$ for MRSA); to linezolid (50\% in MSSA, $80 \%$ for MRSA) and trimethoprim sulfamethoxazole $(18.9 \%$ for MSSA, $60 \%$ for MRSA).

Conclusion: Low MSSA strains (25.3\%) and MRSA (3.3\%) in the communities evaluated, in contrast to studies in health care workers (30\% prevalence for MRSA).

Keywords: Staphylococcus aureus, Community Medicine, Methicillin-Resistant Staphylococcus aureus 


\section{Introducción}

Staphylococcus aureus es un coco gram positivo con alta circulación en el contexto hospitalario y en la comunidad. S. aureus es un reconocido patógeno con la habilidad de adaptarse a diferentes condiciones ambientales, evadir el efecto del tratamiento antimicrobiano y causante de la inadecuada prescripción de antibióticos generando altos costos en su control y alta tasa de mortalidad $(1,2)$.

La presencia de S. aureus ha sido evidenciada en trabajadores de la salud, en comunidad y en pacientes con infecciones invasivas, siendo descritas cepas meticilino resistentes (SAMR), vancomina intermedio (SAVI) y unas pocas vancomicina resistentes (SAVR) (3-7).

El tratamiento de elección en las infecciones por SAMR es la vancomicina intravenosa, sin embargo, otros antibióticos como la clindamicina, trimetoprim sulfametoxazol TMP-SMX, daptomicina y el linezolid han sido probados con similares efectos terapéuticos a la vancomicina, pocos efectos adversos y buena recuperación de los pacientes (8-14).

Varios estudios a nivel mundial han encontrado cepas de $S$. aureus meticilino resistente adquirido en comunidad (SAMR-AC) y cepas adquiridas en el hospital (SAMR-AH), encontrando una prevalencia en general de SAMR de $23 \%$ en Australia, $67 \%$ en Japón, $40 \%$ en el sur del Pacífico, 32\% en USA, y $26 \%$ en Europa. Sosa et al. y Villalobos et al. en Colombia, demostraron la presencia de SAMR-AC en un $60 \%$ de las infecciones usando métodos convencionales y biología molecular en niños y adultos $(15,16)$ y Spirandelli et al. la presencia de SAMR-AH como patógeno nosocomial en Brasil, aislado en un $41.2 \%$ en las neumonías asociadas a ventilador (17).

Finalmente, se reconoce que individuos asintomáticos son un importante reservorio del S. aureus; normalmente como portadores nasales y/o en piel (18), en particular su circulación en trabajadores de la salud con una prevalencia aproximadamente del $30 \%$, pero poco o nada se reconoce sobre la presencia de este microorganismo en otras comunidades, por lo anterior, el objetivo de este estudio es establecer la prevalencia del $S$. aureus aislado de cinco comunidades no relacionadas con el contexto hospitalario e identificar la susceptibilidad a varios antibióticos.

\section{Materiales y Métodos}

Diseño observacional transversal con un muestreo no aleatorio en 150 sujetos de 5 comunidades ( 30 de cada grupo: estudiantes de medicina en entrenamiento clínico (estudiantes entre VI a XII semestre de la Universidad Militar Nueva Granada), amas de casa, manipuladores de alimentos, personas de atención al público y conductores de servicio público), entre los meses de octubre de 2013 y febrero de 2014. Este tipo de poblaciones fueron contempladas por el grupo investigador ante el contacto comunitario que tienen constantemente, lo que permite la circulación del Staphylococcus aureus. Como criterios de inclusión se tuvo que las personas que ingresaran tuvieran como actividad laboral principal las ya mencionadas previamente y como criterios de exclusión el que hubiera recibido antibióticos en los últimos 3 meses. Con respecto al sesgo de selección que se pudiera presentar se controló mediante la elección de los participantes en el estudio con desconocimiento de los mismos por parte del grupo investigador.

Firmado el consentimiento informado y preguntado sobre la convivencia con niños ( $<8$ años), se procedió a tomar muestras de hisopados nasales (19-22) las cuales fueron procesadas en agar sangre (23), posteriormente a las $24 \mathrm{~h}$, se identificaban las colonias de cocos gram positivos beta hemolíticos para realizar la prueba de catalasa; si esta resultaba positiva se procedía a efectuar el test de coagulasa y cultivar en agar sal manitol (24), cada uno de estos procesos se realizaba en cada comunidad estudiada, buscando la presencia del microorganismo objeto de estudio.

Una vez aislado el microorganismo (Staphylococcus aureus), para evaluar la susceptibilidad antibiótica se empleó la técnica de Kirby-Bauer; mediante el sensidisco de cefoxitina se estableció el patrón de resistencia a la meticilina y posteriormente de igual manera se evaluó la resistencia antimicrobiana al linezolid, la clindamicina y el trimetoprim-sulfametoxazol (TMP-SMX) $(8,9,10,25)$.

Para determinar el perfil de sensibilidad a la vancomicina y la concentración inhibitoria mínima se utilizó el método E-test ${ }^{\circledR}$ (Biomerieux) (26). Los resultados se evaluaron de acuerdo con las directrices 2013 del CLSI (Clinical and Laboratory Standards Institute) $(27,28)$.

La incubación para todas las pruebas se realizó a $35^{\circ} \mathrm{C}$ bajo $5 \%$ de $\mathrm{CO}^{2}$ durante 24 horas. En algunos casos para confirmar la biotipificación se realizó API20 Staph ${ }^{\circledR}$ (Biomerieux) o serotipificación con antisueros comerciales (Staphytect plus ${ }^{\circledR}$ Oxoid).

El análisis estadístico se realizó descriptivamente mediante porcentaje de los datos encontrados, correspondientes al número de aislamientos del microorganismo en cuestión, aislamientos de SAMR y posteriormente determinando su susceptibilidad antimicrobiana a los demás antibióticos alternativos de uso clínico.

\section{Resultados}

Se identificaron 43 (28,7\%) aislamientos de Staphylococcus aureus en 150 muestras recolectadas de 5 comunidades, predominando el SAMS en un $88,4 \%$. El número de cepas de $S$. aureus aisladas en el género femenino fue superior en un $16 \%$ a los aislamientos en hombres y solo cinco cepas de SAMR $(12,6 \%)$ sensibles a la vancomicina se aislaron en mujeres (Figura 1). Cuatro de los aislamientos de SAMR se encontraron en personas que convivían con niños (Figura 2). 


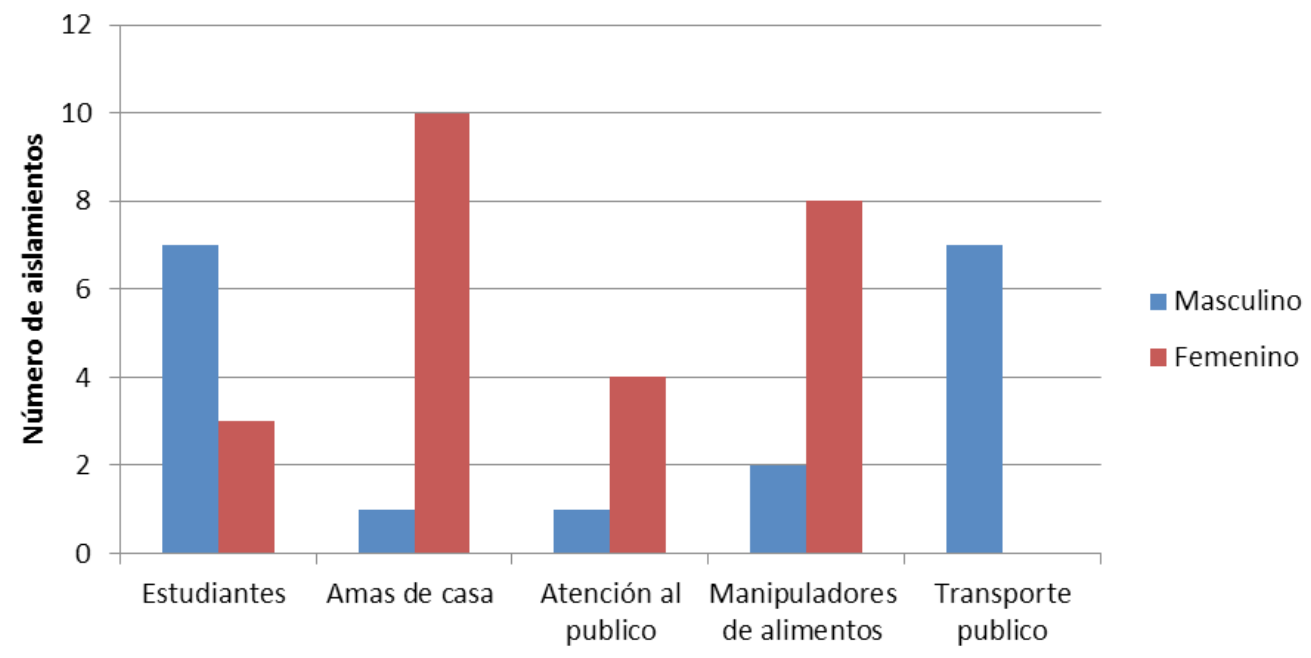

Figura I. Aislamientos de S. aureus según género y comunidad.

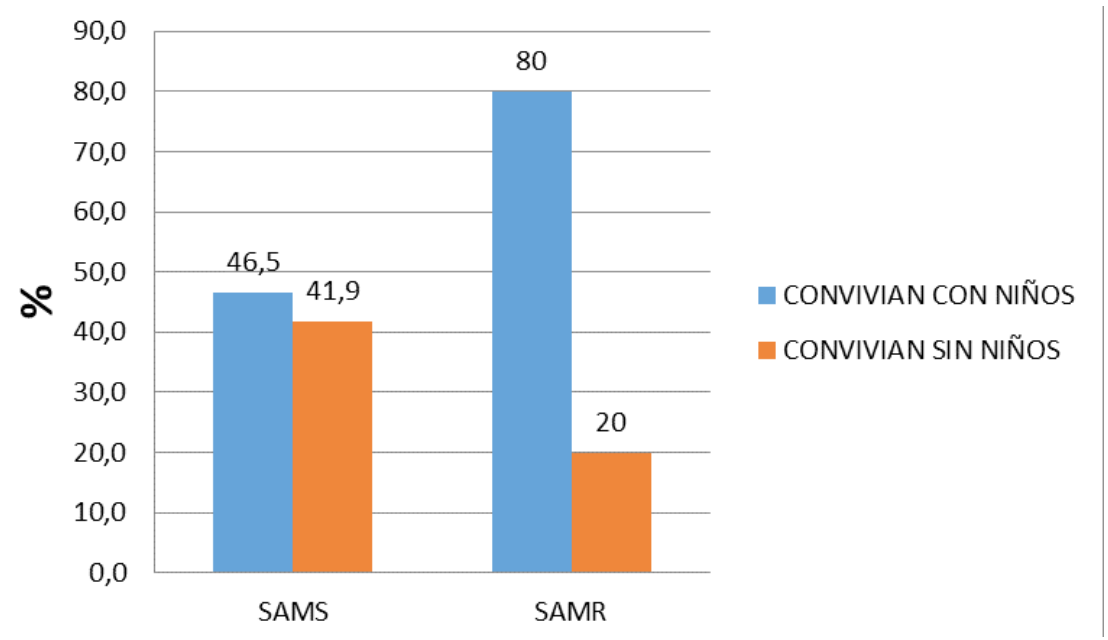

Figura 2. Individuos portadores de cepas de S. aureus con relación a convivencia con niños.

Con respecto al perfil de susceptibilidad, para las cepas de SAMS se encontró resistencia al linezolid y a la clindamicina en un 50\% y 31,6\%, respectivamente. Para las cepas de SAMR, la resistencia a los tres antibióticos probados fue del 60\% al 80\% (Figura 3).

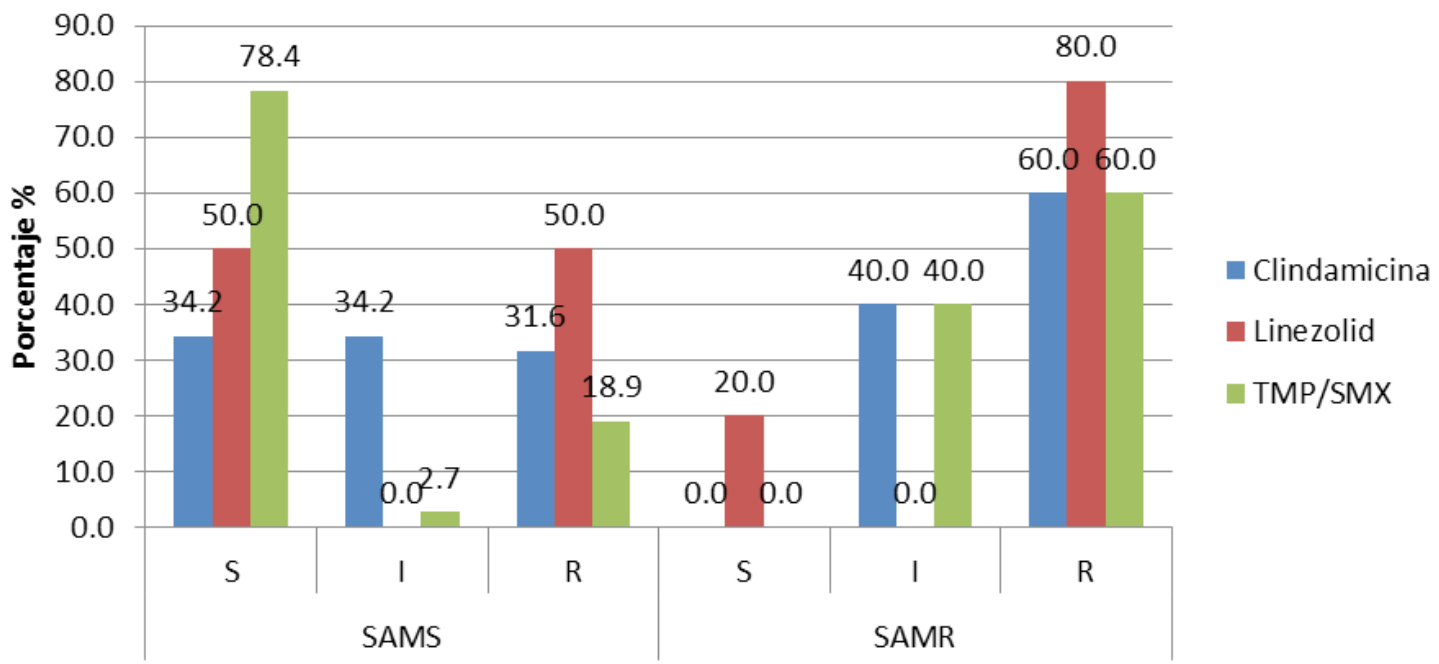

Figura 3. Perfil de susceptibilidad de las cepas de S. aureus aisladas en la comunidad. 


\section{Discusión}

Se describen los aislamientos de Staphylococcus aureus de la cavidad nasal en 5 diferentes comunidades en la ciudad de Bogotá, metrópoli de 7,6 millones de habitantes según datos del Departamento Administrativo Nacional de Estadística de Colombia. Las comunidades evaluadas fueron estudiantes de medicina (entrenamiento básico), amas de casa, personal de atención al público (secretarias, cajeros, vigilantes, tenderos), manipuladores de alimentos y conductores de transporte público. De acuerdo con la distribución del $S$. aureus, se encontró comúnmente en mujeres, esto probablemente se debe a que, en el universo de toma de muestra, las actividades están asociadas en la mayoría de los casos a personas del género femenino, excepto los conductores de transporte público (figura 1).

En un trabajo previo, nuestro grupo encontró 72 (43,9\%) cepas de $S$. aureus aisladas de manos y cavidad nasal de 82 estudiantes de medicina en rotaciones clínicas, $72,2 \%$ fueron identificadas como SAMS, 27,8 \% como meticilino resistentes (SAMR) y cuatro cepas de SAMR mostraron ser vancomicina intermedio (4-8 $\mu \mathrm{g} / \mathrm{mL})$, mientras en el presente estudio los aislamientos de $S$. aureus en general y de cepas meticilino resistentes fueron significativamente menores.

El $43 \%$ de los SAMS fue resistente a clindamicina, 32\% al linezolid y $28 \%$ a TMP/SMX, en contraste a los aislamientos en comunidad donde la resistencia a la clindamicina fue de $31,6 \%$, $50 \%$ al linezolid y 18,9\% al TMP/SMX; siendo las cepas de comunidad más sensibles que los aislamientos de personal en contacto con entornos clínicos a la clindamicina y TMP/SMX, medicamentos frecuentes de segunda línea para el manejo de infecciones por este microorganismo $(29,30)$.

Con respecto a estudios en la comunidad, López- Aguilera et al., evaluaron estudiantes de tercer curso de medicina donde encontró una prevalencia del 34,3\%, y ni un solo aislamiento de SAMR, de manera similar a nuestro estudio (31). Otra población estudiada fue la militar donde Ellis et al. obtuvieron $42 \%$ de aislamientos de $S$. aureus en muestras de cavidad nasal, con un 3,9\% de SAMR y 38\% de SAMS (32). En este mismo grupo poblacional Qu et al., aislaron de 1044 voluntarios un $20 \%$ de $S$. aureus sin encontrar SAMR (33).

Más de 20 mil cepas de fueron recogidas y evaluadas en 56 países a partir de pacientes infectados entre 2004 y 2009 por el Staphylococcus aureus, el 41,2\% eran SAMR y el 58,8\% SAMS. Un total de $4 \%$ de los aislamientos de SAMR y SAMS exhibieron una $\mathrm{CIM} \geq 2 \mu \mathrm{g} / \mathrm{mL}$ a la vancomicina. linezolid, minociclina, tigeciclina; encontrando una disminución global en la susceptibilidad a la vancomicina para los SAMR del 100\% en 2004 al $95,7 \%$ en 2009. Del mismo modo, para los SAMS la susceptibilidad a la vancomicina disminuyó de $100 \%$ en 2004 al 91,1\% en 2009 (4,14). En nuestro trabajo, todas las cepas de SAMR fueron sensibles a la vancomicina, sin embargo, tanto para los SAMS como para los SAMR se destaca la resistencia a los medicamentos alternativos como el linezolid, TMP-SMX y clindamicina.
Cabe hacer énfasis que los pocos aislamientos de SAMR fueron encontrados en personas que convivían con niños (figura 2), lo cual nos sugiere que los niños pudieran ser la fuente de circulación de este tipo de cepas, donde una posible explicación es la selección derivada del mal uso de antibióticos en este tipo de individuos. Esto lo corrobora Hernandez I. et al., quienes en 2003 estudiaron las cepas colonizadoras de la cavidad nasal en niños hospitalizados y niños sanos encontrando un $20 \%$ de aislamientos de $S$. aureus del muestreo total y una resistencia del 25\% a la oxacilina, TMP/SMX y clindamicina (34). Es importante destacar que los niños particularmente los que realizan actividades físicas de contacto están en mayor riesgo de adquirir una infección por cepas de $S$. aureus meticilino resistentes.

Es claro que la presencia de cualquier tipo de cepa de $S$. aureus en una comunidad en particular será motivo de preocupación tomando en cuenta la versatilidad en virulencia y resistencia a los antimicrobianos por parte de este microorganismo (35), por tal razón, varias estrategias sean han considerado para disminuir la circulación de este patógeno, una de ellas, la descolonización general, basada en la mupirocina intranasal, y lavado de manos con clorhexidina. Sin embargo, hasta ahora la mayoría de los estudios que evalúan la descolonización de portadores no encontraron pruebas concluyentes de la eficacia. Algunos reportes indican que la descolonización no redujo la tasa de infección en los portadores de $S$. aureus en menor riesgo de infección, comparado con los pacientes con alto riesgo de infección, donde la descolonización si tuvo un gran impacto (36).

En conclusión, nuestros resultados muestran en general menor prevalencia del $S$. aureus y de cepas meticilino resistente en las comunidades evaluadas comparándolas con estudios en trabajadores de la salud (37) o en estudiantes de medicina en entrenamiento clínico $(38,39)$; al igual que la mayor sensibilidad antimicrobiana de los SAMS a la clindamicina, TMP/SMX y el linezolid. A pesar de los pocos aislamientos de SAMR, se encontró relación de éstos con el contacto de niños, lo cual nos haría sospechar que la población pediátrica es un importante factor para considerar en la circulación de dichos microorganismos.

Debido a la falta de evidencia sólida que permita establecer protocolos de descolonización en trabajadores de la salud (entre ellos los estudiantes de Medicina) y portadores en comunidad, es necesario enfatizar en las medidas de higiene personal, principalmente el lavado de manos, con el fin de reducir al mínimo la circulación de cualquier tipo de cepa de $S$. aureus en los entornos mencionados.

\section{Financiación}

Subvención MED 1360 del Fondo de investigaciones de la Universidad Militar Nueva Granada, Bogotá, Colombia.

\section{Conflicto de interés}

Los autores no tienen ningún conflicto de interés. 


\section{Agradecimientos}

Queremos agradecer a todas las personas que brindaron su apoyo a este estudio, principalmente a Iveth Hernández por su colaboración en la preparación de insumos de laboratorio.

\section{Bibliografía}

1. Matlow A, Morris S. Control of antibiotic-resistant bacteria in the office and clinic. CMAJ 2009;180(10):1021-1024.

2. Plata K, Rosato A, Wegrzyn G. Staphylococcus aureus as an infectious agent: overview of biochemistry and molecular genetics of its pathogenicity. Acta Biochem Polon 2009;56(4):597-612.

3. Paul M, Kariv G, Goldberg E, Raskin M, Shaked H, Hazzan R, et al. Importance of appropriate empirical antibiotic therapy for methicillinresistant Staphylococcus aureus bacteremia. J Antimicrob Chemother 2010;65:2658-2665.

4. Rehm S, Tice A. Staphylococcus aureus: Methicillin-Susceptible S. aureus to Methicillin-Resistant $S$. aureus and Vancomycin-Resistant $S$. aureus. Clin Infect Dis 2010;51(S2):176-182.

5. Cui L, Iwamoto A, Lian J, Neoh H, Maruyama T, Horikawa Y, et al. A Novel Mechanism of Antibiotic Resistance Originating in Vancomycin-Intermediate Staphylococcus aureus. Antimicrob Agents Chemother 2006;50(2):428-238

6. Liu C, Chambers H. Staphylococcus aureus with Heterogeneous Resistance to Vancomycin: Epidemiology, Clinical Significance, and Critical Assessment of Diagnostic Methods. Antimicrob Agents Chemother 2003; 47(10):3040-3045.

7. Sieradzki K, Leski T, Dick J, Borio L, Tomasz A. Evolution of a Vancomycin-Intermediate Staphylococcus aureus Strain In Vivo: Multiple Changes in the Antibiotic Resistance Phenotypes of a Single Lineage of Methicillin-Resistant $S$. aureus under the Impact of Antibiotics Administered for Chemotherapy. J Clin Microbiol 2003;41(4):1687-1693.

8. Frei C, Miller M, Lewis II J, Lawson K, Peddaiahgari R, Talbert R. Retrospective Cohort Study of Hospitalized Adults Treated With Vancomycin or Clindamycin for Methicillin-Resistant Staphylococcus aureus Skin Infections. Clin Ther. 2010;32 (12):2024-2029.

9. Itani K, Dryden M, Bhattacharyya H, Kunkel M, Baruch A, Weigelt J. Efficacy and safety of linezolid versus vancomycin for the treatment of complicated skin and soft-tissue infections proven to be caused by methicillin-resistant Staphylococcus aureus. Am J Surg 2010;199(6):804-816.

10. Balkhair A, Al Muharrmi Z, Darwish L, Farhan H, Sallam M. Treatment of vancomycin-intermediate Staphylococcus aureus (VISA) endocarditis with linezolid. Int J Infect Dis 2010;14:e227-e229.

11. Wang W, Lee S-Y, Chiueh T-S, Lu J-J. Molecular and Phenotypic Characteristics of Methicillin-Resistant and Vancomycin-Intermediate Staphylococcus aureus Isolates from Patients with Septic Arthritis. J Clin Microbiol 2009;47(11):3617-3623.

12. Cui L, Tominaga E, Neoh H, Hiramatsu K. Correlation between Reduced Daptomycin Susceptibility and Vancomycin Resistance in Vancomycin-Intermediate Staphylococcus aureus. Antimicrob Agents Chemother. 2006;50(3):1079-1082.

13. Appleman M, Citron D. Efficacy of vancomycin and daptomycin against Staphylococcus aureus isolates collected over 29 years. Diagnostic Microbiol Infect Dis 2010;66:441-444.
14. Hawser SP, Bouchillon SK, Hoban DJ, Dowzicky M, Babinchak T. Rising incidence of Staphylococcus aureus with reduced susceptibility to vancomycin and susceptibility to antibiotics: a global analysis 2004-2009. Int J Antimicrob Agents 2011;37:219-224.

15. Sosa L, Machuca M, Sosa C, González C. Infecciones por Staphylococcus aureus meticilino resistente en niños en Bucaramanga Colombia. Salud UIS 2010;42: 248-255.

16. Villalobos A, Díaz M, Barrero L, Rivera S, Henriquez D, Villegas M, Robledo C. Tendencias de los fenotipos de resistencia bacteriana en hospitales públicos y privados de alta complejidad de Colombia. Rev Panam Salud Publica.2011;30(6):627-633.

17. Spirandelli K, Mamizuka E, Gontijo P. Methicillin/Oxacillin-resistant Staphylococcus aureus as a hospital and public healt threat in Brazil. Brazilian J Infec Dis 2010;14(1):71-76.

18. Morell E, Balkin D. Methicillin-resistant Staphylococcus aureus: A pervasive pathogen highlights the need for new antimicrobial development. Yale J Biol Med 2010;83:223-233.

19. Cáceres M. Frecuencia de portadores nasales de Staphylococcus aureus resistente a meticilina en personal de salud de hospitales de Nicaragua. Rev Panam Salud Pública 2011;30(6):610-614.

20. Seybold U, Schubert S, Bognera J, Hogardt M. Staphylococcus aureus infection following nasal colonization: an approach to rapid risk stratification in a university healthcare system. J Hosp Infect 2011;79(1):297-301.

21. Rafee Y, Abdel-Haq N, Asmar B, Salimnia T, Vidaillac C, Rybak MJ, et al. Increased prevalence of methicillin-resistant Staphylococcus aureus nasal colonization in household contacts of children with community acquired disease. BMC Infect Dis 2012;75(1):12-45.

22. Creamer E, Dorrian S, Dolan A, Sherlock O, Fitzgerald-Hughes D, Thomas $\mathrm{T}$, et al. When are the hands of healthcare workers positive for meticillin-resistant Staphylococcus aureus? J Hosp Infect 2010;75(1):107-111.

23. Hernández I, Toraño G, González M, González I. Staphylococcus aureus resistente a la meticilina: detección de portadores entre niños hospitalizados y niños sanos de la comunidad. Rev Cubana Med Trop 2003;55(3):153-61.

24. Palavecino E. Métodos recomendados para el estudio de susceptibilidad en Staphylococcus aureus, Staphylococcus coagulasa negativa y Staphylococcus saprophyticus: Nuevos puntos de corte e interpretación de resultados. Rev Chil Infect 2002;19(2):119-124.

25. Mamani E, Luján D, Pajuelo G. Perfil de sensibilidad y resistencia de Staphylococcus aureus. Experiencia en el Hospital Nacional Hipólito Unanue. Annal Fac Med Lima 2006;67(2):120-124

26. Jaramillo S. Prueba Épsilon (Etest). Rev CES Med 1998;12(1):34-41.

27. Clinical and Laboratory Standards Institute. Section 2C Performance standards for antimicrobial susceptibility testing; twenty-third informational supplement. 2013; 33(1):72-89.

28. Malhotra-Kumar S, Haccuria K, Michiels M, Ieven M, Poyart C, Hryniewicz W, et al. MINIREVIEW. Current Trends in Rapid Diagnostics for Methicillin-Resistant Staphylococcus aureus and Glycopeptide-Resistant Enterococcus Species. J Clin Microbiol 2008;46(5):1577-1587.

29. Méndez I, Holguín D, Pachón D, Africano F, Gonzalez I, Rojas N. Prevalence and antimicrobial susceptibility of Staphylococcus aureus methicilin resistant isolated from medical students. Rev CES Med 2013;27(1):21-30.

30. Figueroa A, Arteaga M, Aguirre J, et al. Infections by methicillin-resistant Staphylococcus aureus Community-acquired (MRSA-CA) in the pediatric population in two hospitals in Popayan, Colombia. Rev Fac Cienc de la Salud. Univ del Cauca 2010; 12 (4): 8-13. 
31. López-Aguilera S, Goñi-Yeste M, Barrado L, et al. Colonización nasal por Staphylococcus aureus en estudiantes de medicina: importancia en la transmisión hospitalaria. Enferm Infecc Microbiol Clin. 2013;31(8):500-505.

32. Ellis M, Griffith M, Jorgensen J, et al. Presence and Molecular Epidemiology of Virulence Factors in Methicillin-Resistant Staphylococcus aureus Strains Colonizing and Infecting Soldiers. J. Clin. Microbiol. 2009;47(4):940-945.

33. Qu F, Cui E, Guo T, et al. Nasal Colonization of and Clonal Transmission of Methicillin-Susceptible Staphylococcus aureus among Chinese Military Volunteers. J. Clin. Microbiol. 2010;48(1):64-69.

34. Hernández I, Toraño G, González M, et al. Staphylococcus aureus resistente a la meticilina: detección de portadores entre niños hospitalizados y niños sanos de la comunidad. Rev Cubana Med Trop 2003;55(3):153-61.

35. Fosch S, Yones C, Trossero M, Grosso O, Nepote A. Portación nasal de Staphylococcus aureus en individuos de la comunidad: factores epidemiológicos. Acta Bioquím Clín Latinoam 2012;46(1): 59-67.

36. Lucet J, Regnier B, Screening and Decolonization: Does Methicillin-Susceptible Staphylococcus aureus Hold Lessons for Methicillin-Resistant $S$. aureus? Clin Infec Dis 2010;51(5):585-590.

37. 37. Espinosa C, Romero M, Rincón G, Bohórquez M, Arámbula A. Portadores nasales de Staphylococcus aureus en personal que labora en un hospital de Santander. Salud UIS 2011;43(2):111-117.

38. Méndez I, Calixto O, Becerra W, Vásquez J, Bravo J, Pachón D. Microorganismos presentes en fonendoscopios, manos, cavidad oral y nasal de estudiantes de una facultad de Medicina. Rev MED. 2012;20(1):90-100.

39. Gandia J, Benjumea Y, Mangones L, Villacob K, Sánchez L, Mosquera E. Prevalencia de Staphylococcus aureus meticilino resistente en estudiantes de medicina en la Universidad del Sinú. Reporte in Primer Encuentro nacional de semilleros de de investigación - facultades de Medicina, 2012 july 25-27, Bogotá, Colombia. 